\title{
IMPLICIT FRACTIONAL DIFFERENTIAL EQUATION INVOLVING $\psi$-CAPUTO WITH BOUNDARY CONDITIONS
}

\author{
BOUTIARA ABDELLATIF ${ }^{1, a}$ AND MAAMAR BENBACHIR ${ }^{2, b}$ \\ ${ }^{1}$ Laboratory of Mathematics And Applied Sciences, University of Ghardaia, 47000. Algeria. \\ ${ }^{a}$ E-mail: boutiara_a@yahoo.com \\ ${ }^{2}$ Faculty of Sciences, Saad Dahlab University, Blida, Algeria. \\ ${ }^{b}$ E-mail: mbenbachir2001@gmail.com \\ \|\|
}

\section{Abstract}

In this research article, we discuss the existence results and uniqueness of solutions for a class of boundary value problems of fractional differential equations with the $\psi$-Caputo fractional derivative. The reasoning is mainly based upon different types of classical fixed point theory such as the Banach contraction principle and Krasnoselskii's fixed point theorem. Besides, the Ulam-Hyers result is addressed for the proposed problem. We illustrate our main findings, with a particular case example included to show the applicability of our outcomes.

\section{Introduction}

Fractional calculus generalizes the integer-order integration and differentiation concepts to an arbitrary(real or complex) order. Fractional calculus is one of the most emerging areas of investigation. The fractional differential operators are used to model many biological and physical phenomena, mathematical modeling of engineering, etc. in a much better form as compared to ordinary differential operators, which are local. To get a couple of developments about the theory of fractional differential equations, one can allude to the monographs of Hilfer [17], Kilbas et al [22], Miller and Ross [23], Oldham [25], Pudlubny [26], Sabatier et al [28], Tarasov [31] and the references therein.

Received March 5, 2021.

AMS Subject Classification: 34B15, 34B18, 26A33, 34A12.

Key words and phrases: Implicit fractional differential equations, $\psi$-Caputo fractional derivative, fractional $\psi$-integral, Ulam-Hyers stability, Boundary value problem, fixed point theorem. 
At the present day, there are numerous results on the existence and uniqueness of solutions for fractional differential equations. For greater details, the readers are cited the previous research [22, 10, 15, 12, 4, 8] and the references therein. however, due to the fact that in lots of conditions, which include nonlinear analysis and optimization, locating the exact solution of differential equations is almost tough or impossible, we don't forget approximate solutions. it is essential to observe that only stable approximate solutions are proper. various approaches of stability analysis are adopted for this reason. The HU-type stability concept has been taken into consideration in the severa literature. The said stability analysis is an clean and easy manner on this regard. This type idea of stability become formulated for the primary time by means of Ulam [32], and then the next year it become elaborated with the aid of Hyers [18].

Inside the starting, this concept became implemented to ordinary differential equations after which extended to fractional diferential equation FDEs. We refer the readers to [19, 20, 21, 24, 9, 27].

In this paper deals with the existence and uniqueness of solutions for boundary-value problem of the nonlinear $\psi$-Caputo fractional differential equations

$$
\left\{\begin{array}{l}
{ }^{C} \mathcal{D}_{a^{+}}^{\alpha, \psi} u(t)=f\left(t, u(t),{ }^{C} \mathcal{D}_{a^{+}}^{\alpha, \psi} u(t)\right), \quad t \in J:=[a, T] \\
u(T)=\lambda u(\eta)
\end{array}\right.
$$

where ${ }^{C} \mathcal{D}_{a^{+}}^{\alpha ; \psi}$ is the $\psi$-Caputo fractional derivative of order $\alpha \in(0,1]$, $f:[a, T] \times \mathbb{R} \longrightarrow \mathbb{R}$ is a given continuous function. $\lambda$ is real constant and $\eta \in(a, T)$.

Here is a brief outline of the paper. The Section 2 provides the definitions and preliminary results that we will need to prove our main results and present an auxiliary lemma that provides solution representation for the solutions of Problem (1). In Section 3, we establish existence and uniqueness for fractional differential equations involving $\psi$-Caputo fractional differential operator. In Section 4, we discuss some types of fractional Ulam stability. In Section 5, we give an example to illustrate the obtained results. 


\section{Preliminaries and Lemmas}

In this section, we introduce some notations and definitions of fractional calculus and present preliminary results needed in our proofs later.

Let $C(J, \mathbb{R})$ the space of real and continuous functions with the norm

$$
\|u\|_{\infty}=\sup \{\|u(t)\|: \quad t \in J\} .
$$

Let $L^{1}(J, \mathbb{R})$ be the Banach space of Lebesgue integrable functions $u: J \rightarrow \mathbb{R}$, equipped with the norm

$$
\|u\|_{L^{1}}=\int_{J}|u(t)| d t
$$

We begin by defining $\psi$-Riemann-Liouville fractional integrals and derivatives. In what follows,

Definition 1 ([4] $)$. For $\alpha>0$, the left-sided $\psi$-Riemann-Liouville fractional integral of order $\alpha$ for an integrable function $u: \mathrm{J} \longrightarrow \mathbb{R}$ with respect to another function $\psi: \mathrm{J} \longrightarrow \mathbb{R}$ that is an increasing differentiable function such that $\psi^{\prime}(t) \neq 0$, for all $t \in \mathrm{J}$ is defined as follows

$$
\mathcal{I}_{a^{+}}^{\alpha ; \psi} u(t)=\frac{1}{\Gamma(\alpha)} \int_{a}^{t} \psi^{\prime}(s)(\psi(t)-\psi(s))^{\alpha-1} u(s) \mathrm{ds},
$$

where $\Gamma$ is the classical Euler Gamma function.

Definition 2 ([4] $)$. Let $n \in \mathbb{N}$ and let $\psi, u \in C^{n}(\mathrm{~J}, \mathbb{R})$ be two functions such that $\psi$ is increasing and $\psi^{\prime}(t) \neq 0$, for all $t \in \mathrm{J}$. The left-sided $\psi$ Riemann-Liouville fractional derivative of a function $u$ of order $\alpha$ is defined by

$$
\begin{aligned}
\mathcal{D}_{a^{+}}^{\alpha ; \psi} u(t) & =\left(\frac{1}{\psi^{\prime}(t)} \frac{d}{d t}\right)^{n} \mathcal{I}_{a^{+}}^{n-\alpha ; \psi} u(t) \\
& =\frac{1}{\Gamma(n-\alpha)}\left(\frac{1}{\psi^{\prime}(t)} \frac{d}{d t}\right)^{n} \int_{a}^{t} \psi^{\prime}(s)(\psi(t)-\psi(s))^{n-\alpha-1} u(s) \mathrm{d} \mathrm{s},
\end{aligned}
$$

where $n=[\alpha]+1$.

Definition $3([4])$. Let $n \in \mathbb{N}$ and let $\psi, u \in C^{n}(\mathrm{~J}, \mathbb{R})$ be two functions such that $\psi$ is increasing and $\psi^{\prime}(t) \neq 0$, for all $t \in \mathrm{J}$. The left-sided $\psi$-Caputo 
fractional derivative of $u$ of order $\alpha$ is defined by

$$
{ }^{C} \mathcal{D}_{a^{+}}^{\alpha ; \psi} u(t)=\mathcal{I}_{a^{+}}^{n-\alpha ; \psi}\left(\frac{1}{\psi^{\prime}(t)} \frac{d}{d t}\right)^{n} u(t),
$$

where $n=[\alpha]+1$ for $\alpha \notin \mathbb{N}, n=\alpha$ for $\alpha \in \mathbb{N}$.

To simplify notation, we will use the abbreviated symbol

$$
u_{\psi}^{[n]}(t)=\left(\frac{1}{\psi^{\prime}(t)} \frac{d}{d t}\right)^{n} u(t) .
$$

From the definition, it is clear that

$$
{ }^{C} \mathcal{D}_{a^{+}}^{\alpha ; \psi} u(t)=\left\{\begin{array}{cc}
\int_{a}^{t} \frac{\psi^{\prime}(s)(\psi(t)-\psi(s))^{n-\alpha-1}}{\Gamma(n-\alpha)} u_{\psi}^{[n]}(s) \mathrm{ds} & , \text { if } \alpha \notin \mathbb{N}, \\
u_{\psi}^{[n]}(t) & , \text { if } \alpha \in \mathbb{N} .
\end{array}\right.
$$

We note that if $u \in C^{n}(\mathrm{~J}, \mathbb{R})$ the $\psi$-Caputo fractional derivative of order $\alpha$ of $u$ is determined as

$$
{ }^{C} \mathcal{D}_{a^{+}}^{\alpha ; \psi} u(t)=\mathcal{D}_{a^{+}}^{\alpha ; \psi}\left[u(t)-\sum_{k=0}^{n-1} \frac{u_{\psi}^{[k]}(a)}{k !}(\psi(t)-\psi(a))^{k}\right] .
$$

(see, for instance, [4, Theorem 3]).

Lemma 1 ([6] $)$. Let $\alpha, \beta>0$, and $u \in L^{1}(\mathrm{~J}, \mathbb{R})$. Then

$$
\mathcal{I}_{a^{+}}^{\alpha ; \psi} \mathcal{I}_{a^{+}}^{\beta ; \psi} u(t)=\mathcal{I}_{a^{+}}^{\alpha+\beta ; \psi} u(t) \text {, a.e. } t \in \mathrm{J} .
$$

In particular, if $u \in \mathcal{C}(\mathrm{J}, \mathbb{R})$, then $\mathcal{I}_{a^{+}}^{\alpha ; \psi} \mathcal{I}_{a^{+}}^{\beta ; \psi} u(t)=\mathcal{I}_{a^{+}}^{\alpha+\beta ; \psi} u(t), t \in \mathrm{J}$.

Lemma $2([6])$. Let $\alpha>0$, The following holds:

If $u \in \mathcal{C}(\mathrm{J}, \mathbb{R})$ then

$$
\begin{gathered}
{ }^{C} \mathcal{D}_{a^{+}}^{\alpha ; \psi} \mathcal{I}_{a^{+}}^{\alpha ; \psi} u(t)=u(t), t \in \mathrm{J} . \\
\text { If } u \in C^{n}(\mathrm{~J}, \mathbb{R}), n-1<\alpha<n . \text { Then } \\
\mathcal{I}_{a^{+}}^{\alpha ; \psi}{ }^{C} \mathcal{D}_{a^{+}}^{\alpha ; \psi} u(t)=u(t)-\sum_{k=0}^{n-1} \frac{u_{\psi}^{[k]}(a)}{k !}[\psi(t)-\psi(a)]^{k}, \quad t \in \mathrm{J} .
\end{gathered}
$$


Lemma $3([6])$. Let $t>a, \alpha \geq 0$, and $\beta>0$. Then

- $\mathcal{I}_{a^{+}}^{\alpha ; \psi}(\psi(t)-\psi(a))^{\beta-1}=\frac{\Gamma(\beta)}{\Gamma(\beta+\alpha)}(\psi(t)-\psi(a))^{\beta+\alpha-1}$,

- ${ }^{C} \mathcal{D}_{a^{+}}^{\alpha ; \psi}(\psi(t)-\psi(a))^{\beta-1}=\frac{\Gamma(\beta)}{\Gamma(\beta-\alpha)}(\psi(t)-\psi(a))^{\beta-\alpha-1}$,

- ${ }^{C} \mathcal{D}_{a^{+}}^{\alpha ; \psi}(\psi(t)-\psi(a))^{k}=0$, for all $k \in\{0, \ldots, n-1\}, n \in \mathbb{N}$.

\section{Main Results}

Before starting and proving our main result we introduce the following auxiliary lemma.

Lemma 4. Let $0<\alpha<1, \rho>0$ and $w \in \mathcal{C}(J, \mathbb{R})$. Then the linear antiperiodic boundary value problem

$$
\begin{aligned}
{ }^{C} \mathcal{D}^{\alpha, \psi} u(t) & =\sigma(t), \quad t \in J, \\
u(T) & =\lambda u(\eta)
\end{aligned}
$$

has a unique solution defined by

$$
\begin{aligned}
u(t)= & \frac{1}{\Gamma(\alpha)} \int_{a}^{t} \psi^{\prime}(s)(\psi(t)-\psi(s))^{\alpha-1} \sigma(t) d s \\
& +\frac{1}{\Lambda}\left\{\frac{\lambda}{\Gamma(\alpha)} \int_{a}^{\eta} \psi^{\prime}(s)(\psi(\eta)-\psi(s))^{\alpha-1} \sigma(t) d s\right. \\
& \left.+\frac{1}{\Gamma(\alpha)} \int_{a}^{T} \psi^{\prime}(s)(\psi(T)-\psi(s))^{\alpha-1} \sigma(t) d s\right\}
\end{aligned}
$$

Proof. Assume $u$ satisfies (44). Then Lemma 2 implies that

$$
u(t)=\mathcal{I}^{\alpha ; \psi} \sigma(t)+c_{1} .
$$

The condition (41) implies that

$$
\begin{aligned}
& u(T)=\mathcal{I}^{\alpha ; \psi} \sigma(T)+c_{1} \\
& u(\eta)=\mathcal{I}^{\alpha ; \psi} \sigma(\eta)+c_{1} .
\end{aligned}
$$

Thus,

$$
\left.c_{1}(1-\lambda)=\lambda \mathcal{I}^{\alpha ; \psi} \sigma(\eta)\right)-\mathcal{I}^{\alpha ; \psi} \sigma(T)
$$


Consequently,

$$
\left.c_{1}=\frac{1}{\Lambda}\left\{\lambda \mathcal{I}^{\alpha ; \psi} \sigma(\eta)\right)-\mathcal{I}^{\alpha ; \psi} \sigma(T)\right\}
$$

Where,

$$
\Lambda=(1-\lambda)
$$

Finally, we obtain the solution in the equation (5).

Lemma 5. Assume that $f: J \times \mathbb{R} \times \mathbb{R} \rightarrow \mathbb{R}$ is continuous. A function $u(t)$ solves the problem (11) if and only if it is a fixed-point of the operator $\mathcal{G}: \mathcal{C}(J, \mathbb{R}) \rightarrow \mathcal{C}(J, \mathbb{R})$ defined by

$$
\begin{aligned}
\mathcal{G} u(t)= & \frac{1}{\Gamma(\alpha)} \int_{a}^{t} \psi^{\prime}(s)(\psi(t)-\psi(s))^{\alpha-1} f\left(s, u(s),{ }^{C} \mathcal{D}_{a^{+}}^{\alpha ; \psi} u(s)\right) d s \\
& +\frac{1}{\Lambda}\left\{\frac{\lambda}{\Gamma(\alpha)} \int_{a}^{\eta} \psi^{\prime}(s)(\psi(\eta)-\psi(s))^{\alpha-1} f\left(s, u(s),{ }^{C} \mathcal{D}_{a^{+}}^{\alpha ; \psi} u(s)\right) d s\right. \\
& \left.+\frac{1}{\Gamma(\alpha)} \int_{a}^{T} \psi^{\prime}(s)(\psi(T)-\psi(s))^{\alpha-1} f\left(s, u(s),{ }^{C} \mathcal{D}_{a^{+}}^{\alpha ; \psi} u(s)\right) d s\right\} .
\end{aligned}
$$

In the following subsections, we establish the existence and uniqueness of solutions for the boundary value problem (1) by applying a variety of fixed point theorems.

\subsection{Uniqueness result via banach fixed point theorem}

Theorem 1. Assume that $f: J \times \mathbb{R} \times \mathbb{R} \rightarrow \mathbb{R}$ be a continuous function satisfying the Lipschitz condition:

(H1) There exists $L_{1}>0$ and $0<L_{2}<1$ such that:

$$
\left|f\left(t, u_{1}, u_{2}\right)-f\left(t, v_{1}, v_{2}\right)\right| \leq L_{1}\left|u_{1}-v_{1}\right|+L_{2}\left|u_{2}-v_{2}\right|
$$

for $t \in J$ and every $u_{i}, v_{i} \in \mathbb{R},(i=1,2)$ If

$$
\begin{aligned}
\Lambda_{1} & =\frac{L_{1}}{1-L_{2}}\left\{\frac{(\psi(T)-\psi(s))^{\alpha}}{\Gamma(\alpha+1)}+\frac{1}{\Lambda}\left\{\frac{\lambda(\psi(\eta)-\psi(s))^{\alpha}}{\Gamma(\alpha+1)}+\frac{(\psi(T)-\psi(s))^{\alpha}}{\Gamma(\alpha+1)}\right\}\right\} \\
& <1
\end{aligned}
$$

then the boundary value problem (11) has a unique solution on J. 
Proof. In the first step, we prove that $\mathcal{G B}_{r} \subseteq \mathcal{B}_{r}$ where the operator $\mathcal{G}$ : $\mathcal{C}(J, \mathbb{R}) \rightarrow \mathcal{C}(J, \mathbb{R})$ defined by (77) and

$$
\mathcal{B}_{r}=\{u \in \mathcal{C}(J, \mathbb{R}),\|u\| \leq r\}
$$

with choose $r \geq \frac{\Lambda_{2}}{1-\Lambda_{1}}$, where $\Lambda_{1}<1$ and

$$
\Lambda_{2}=\frac{\mu}{1-L_{2}}\left\{\frac{(\psi(T)-\psi(s))^{\alpha}}{\Gamma(\alpha+1)}+\frac{1}{\Lambda}\left\{\frac{\lambda(\psi(\eta)-\psi(s))^{\alpha}}{\Gamma(\alpha+1)}+\frac{(\psi(T)-\psi(s))^{\alpha}}{\Gamma(\alpha+1)}\right\}\right\}
$$

and $\sup l \in J|f(t, 0,0)|:=\mu<\infty$. Set $\mathcal{K}_{u}(t):=f\left(t, u(t),{ }^{C} \mathcal{D}_{a^{+}}^{\alpha \rho} u(t)\right)$. For any $u \in \mathcal{S}_{r}$, we have

$$
\begin{aligned}
|\mathcal{G} u(t)| \leq & \sup _{t \in J}|\mathcal{G} u(t)| \\
\leq & \sup _{t \in J}\left\{\frac{1}{\Gamma(\alpha)} \int_{a}^{t} \psi^{\prime}(s)(\psi(t)-\psi(s))^{\alpha-1}\left|\mathcal{K}_{u}(s)\right| d s\right. \\
& +\frac{1}{\Lambda}\left\{\frac{\lambda}{\Gamma(\alpha)} \int_{a}^{\eta} \psi^{\prime}(s)(\psi(\eta)-\psi(s))^{\alpha-1}\left|\mathcal{K}_{u}(s)\right| d s\right. \\
& \left.\left.+\frac{1}{\Gamma(\alpha)} \int_{a}^{T} \psi^{\prime}(s)(\psi(T)-\psi(s))^{\alpha-1}\left|\mathcal{K}_{u}(s)\right| d s\right\}\right\} .
\end{aligned}
$$

From (H1), we get

$$
\begin{aligned}
\left|\mathcal{K}_{u}(s)\right| & =\left|f\left(s, u(s),{ }^{C} \mathcal{D}_{a^{+}}^{\alpha ; \psi} u(s)\right)\right| \\
& \leq\left|f\left(s, u(s),{ }^{C} \mathcal{D}_{a^{+}}^{\alpha ; \psi} u(\tau)\right)-f(s, 0,0)\right|+|f(\tau, 0,0)| \\
& \leq L_{1}|u(s)|+L_{2}\left|{ }^{C} \mathcal{D}_{a^{+}}^{\alpha, \psi} u(s)\right|+\mu \\
& =L_{1} r+L_{2}\left|\mathcal{K}_{u}(s)\right|+\mu
\end{aligned}
$$

which gives

$$
\left|\mathcal{K}_{u}(s)\right| \leq \frac{\left(L_{1} r+\mu\right)}{1-L_{2}}
$$

Therefore,

$$
\begin{aligned}
|\mathcal{G} u(t)| \leq & \sup _{t \in J}\left\{\frac{\left(L_{1} r+\mu\right)}{1-L_{2}} \frac{1}{\Gamma(\alpha)} \int_{a}^{t} \psi^{\prime}(s)(\psi(t)-\psi(s))^{\alpha-1} d s\right. \\
& +\frac{\left(L_{1} r+\mu\right)}{1-L_{2}} \frac{1}{\Lambda}\left\{\frac{\lambda}{\Gamma(\alpha)} \int_{a}^{\eta} \psi^{\prime}(s)(\psi(\eta)-\psi(s))^{\alpha-1} d s\right.
\end{aligned}
$$




$$
\begin{gathered}
\left.\left.\quad+\frac{\left(L_{1} r+\mu\right)}{1-L_{2}} \frac{1}{\Gamma(\alpha)} \int_{a}^{T} \psi^{\prime}(s)(\psi(T)-\psi(s))^{\alpha-1} d s\right\}\right\} \\
\leq \frac{\left(L_{1} r+\mu\right)}{1-L_{2}}\left\{\frac{(\psi(T)-\psi(s))^{\alpha}}{\Gamma(\alpha+1)}+\frac{1}{\Lambda}\left\{\frac{\lambda(\psi(\eta)-\psi(s))^{\alpha}}{\Gamma(\alpha+1)}+\frac{(\psi(T)-\psi(s))^{\alpha}}{\Gamma(\alpha+1)}\right\}\right\} \\
=\frac{L_{1} r}{1-L_{2}}\left\{\frac{(\psi(T)-\psi(s))^{\alpha}}{\Gamma(\alpha+1)}+\frac{1}{\Lambda}\left\{\frac{\lambda(\psi(\eta)-\psi(s))^{\alpha}}{\Gamma(\alpha+1)}+\frac{(\psi(T)-\psi(s))^{\alpha}}{\Gamma(\alpha+1)}\right\}\right\} \\
+\frac{\mu}{1-L_{2}}\left\{\frac{(\psi(T)-\psi(s))^{\alpha}}{\Gamma(\alpha+1)}+\frac{1}{\Lambda}\left\{\frac{\lambda(\psi(\eta)-\psi(s))^{\alpha}}{\Gamma(\alpha+1)}+\frac{(\psi(T)-\psi(s))^{\alpha}}{\Gamma(\alpha+1)}\right\}\right\} \\
=\Lambda_{1} r+\Lambda_{2}<r \quad\|\mathcal{G} u\|<r
\end{gathered}
$$

which implies that $\mathcal{G} u \in \mathcal{B}_{r}$. Moreover, by (7), and Lammas 2, 3 we obtain

$$
{ }^{C} \mathcal{D}_{a^{+}}^{\alpha \cdot \rho} \mathcal{G} u(t)={ }^{C} \mathcal{D}_{a^{+}}^{\alpha ; \rho} \mathcal{I}_{a^{+}}^{\alpha \rho} \mathcal{K}_{u}(t)=\mathcal{K}_{u}(t)
$$

since $\mathcal{K}_{u}(\cdot)$ is continuous on $J$, the operator ${ }^{C} \mathcal{D}_{a^{+}}^{\alpha \rho} \mathcal{G} u(t)$ is continuous on $J$, that is $\mathcal{G B}_{r} \subseteq \mathcal{B}_{r}$ Next, we apply the Banach fixed point theorem to prove that $\mathcal{G}$ has a fixed point. Indeed, it enough to show that $\mathcal{G}$ is contraction map. Let $u_{1}, u_{2} \in \mathcal{C}(J, \mathbb{R})$ and for $t \in J$. Then, we have

$$
\begin{aligned}
\left|\mathcal{G} u_{1}(t)-\mathcal{G} u_{2}(t)\right| \leq & \left\{\frac{1}{\Gamma(\alpha)} \int_{a}^{t} \psi^{\prime}(s)(\psi(t)-\psi(s))^{\alpha-1}\left|\mathcal{K}_{u_{1}}(s)-\mathcal{K}_{u_{2}}(s)\right| d s\right. \\
& +\frac{1}{\Lambda}\left\{\frac{\lambda}{\Gamma(\alpha)} \int_{a}^{\eta} \psi^{\prime}(s)(\psi(\eta)-\psi(s))^{\alpha-1}\left|\mathcal{K}_{u_{1}}(s)-\mathcal{K}_{u_{2}}(s)\right| d s\right. \\
& \left.\left.+\frac{1}{\Gamma(\alpha)} \int_{a}^{T} \psi^{\prime}(s)(\psi(T)-\psi(s))^{\alpha-1}\left|\mathcal{K}_{u_{1}}(s)-\mathcal{K}_{u_{2}}(s)\right| d s\right\}\right\}
\end{aligned}
$$

by $\left(\mathrm{H}_{1}\right)$, we get

$$
\begin{aligned}
\left|\mathcal{K}_{u_{1}}(s)-\mathcal{K}_{u_{2}}(s)\right| & =\left|f\left(s, u_{1}(s),{ }^{C} \mathcal{D}_{a^{+}}^{\alpha ; \psi} u_{1}(s)\right)-f\left(s, u_{1}(s),{ }^{C} \mathcal{D}_{a^{+}}^{\alpha ; \psi} u_{2}(s)\right)\right| \\
& \leq L_{1}\left|u_{1}-u_{2}\right|+L_{2}\left|{ }^{C} \mathcal{D}_{a^{+}}^{\alpha ; \psi} u_{1}(s)-{ }^{C} \mathcal{D}_{a^{+}}^{\alpha ; \psi} u_{2}(s)\right| \\
& =L_{1}\left|u_{1}-u_{2}\right|+L_{2}\left|\mathcal{K}_{u_{1}}(s)-\mathcal{K}_{u_{2}}(s)\right|
\end{aligned}
$$

which implies

$$
\left|\mathcal{K}_{u_{1}}(s)-\mathcal{K}_{u_{2}}(s)\right| \leq \frac{L_{1}}{1-L_{2}}\left|u_{1}-u_{2}\right|
$$


Then

$$
\begin{aligned}
\left\|\mathcal{G} u_{1}-\mathcal{G} u_{2}\right\| \leq & \frac{L_{1}}{1-L_{2}}\left\{\frac{(\psi(T)-\psi(s))^{\alpha}}{\Gamma(\alpha+1)}\right. \\
& \left.+\frac{1}{\Lambda}\left\{\frac{\lambda(\psi(\eta)-\psi(s))^{\alpha}}{\Gamma(\alpha+1)}+\frac{(\psi(T)-\psi(s))^{\alpha}}{\Gamma(\alpha+1)}\right\}\right\}\left\|u_{1}-u_{2}\right\| .
\end{aligned}
$$

Consequently, $\left\|\mathcal{G} u_{1}-\mathcal{G} u_{2}\right\| \leq \Lambda_{1}\left\|u_{1}-u_{2}\right\|$. Since $\Lambda_{1}<1$, the operator $\mathcal{G}$ is contraction mapping. Hence, we deduce by Banach contraction mapping principle that the operator $\mathcal{G}$ has a unique fixed point, which corresponds to a unique solution of the problem in Equation (1) on $J$. The proof is completed.

\subsection{Existence result via Kransnoselskii's fixed point theorem}

In the next existence result, we apply Krasnoselskii fixed point theorem [28].

Theorem 2. Assume that $\left(H_{1}\right)$ holds. If

$$
\begin{aligned}
\Delta & :=\frac{L_{1}}{1-L_{2}}\left\{\frac{(\psi(T)-\psi(s))^{\alpha}}{\Gamma(\alpha+1)}+\frac{1}{\Lambda}\left\{\frac{\lambda(\psi(\eta)-\psi(s))^{\alpha}}{\Gamma(\alpha+1)}+\frac{(\psi(T)-\psi(s))^{\alpha}}{\Gamma(\alpha+1)}\right\}\right\} \\
& <1
\end{aligned}
$$

then the problem (11) has at least one solution on $J$.

Proof. Consider the operator $\mathcal{G}: \mathcal{C}(J, \mathbb{R}) \rightarrow \mathcal{C}(J, \mathbb{R})$ defined by (77). Define the ball

$$
\mathcal{B}_{r_{0}}:=\left\{u \in \mathcal{C}(J, \mathbb{R}):\|u\| \leq r_{0}\right\}
$$

Now we subdivide the operator $\mathcal{G}$ into two operators $\mathcal{G}_{1}$ and $\mathcal{G}_{2}$ on $\mathcal{B}_{r_{0}}$ defined by

and

$$
\begin{aligned}
\mathcal{G}_{1} u(t)= & \frac{1}{\Lambda}\left\{\frac{\lambda}{\Gamma(\alpha)} \int_{a}^{\eta} \psi^{\prime}(s)(\psi(\eta)-\psi(s))^{\alpha-1} \mathcal{K}_{u}(s) d s\right. \\
& \left.+\frac{1}{\Gamma(\alpha)} \int_{a}^{T} \psi^{\prime}(s)(\psi(T)-\psi(s))^{\alpha-1} \mathcal{K}_{u}(s) d s\right\}
\end{aligned}
$$




$$
\mathcal{G}_{2} u(t)=\frac{1}{\Gamma(\alpha)} \int_{a}^{t} \psi^{\prime}(s)(\psi(t)-\psi(s))^{\alpha-1} \mathcal{K}_{u}(s) d s .
$$

Taking into account that $\mathcal{G}_{1}$ and $\mathcal{G}_{2}$ are defined on $\mathcal{B}_{r_{0}}$, and for any $u \in$ $\mathcal{C}(J, \mathbb{R})$,

$$
\mathcal{G} u(t)=\mathcal{G}_{1} u(t)+\mathcal{G}_{2} u(t), \quad t \in J .
$$

The proof will be divided into several steps:

Step 1: $\mathcal{G}_{1} u_{1}+\mathcal{G}_{2} u_{2} \in \mathcal{B}_{r_{0}}$ for every $u_{1}, u_{2} \in \mathcal{B}_{r_{0}}$. For $u_{1} \in \mathcal{B}_{r_{0}}$ and using the same arguments in (8), we get

$$
\left|\mathcal{K}_{u_{1}}(\tau)\right| \leq \frac{\left(L_{1} r_{0}+\mu\right)}{1-L_{2}}
$$

Similarly, for $u_{2} \in \mathcal{B}_{r_{0}}$, we obain

$$
\left|\mathcal{K}_{u_{2}}(\tau)\right| \leq \frac{\left(L_{1} r_{0}+\mu\right)}{1-L_{2}}
$$

Now, for $u_{1}, u_{2} \in \mathcal{B}_{r_{0}}$ and $t \in J$, we have

$$
\begin{aligned}
\left|\mathcal{G}_{1} u_{1}(t)+\mathcal{G}_{2} u_{2}(t)\right| \leq & \left|\mathcal{G}_{1} u_{1}(t)\right|+\left|\mathcal{G}_{2} u_{2}(t)\right| \\
\leq & \left\{\frac{1}{\Gamma(\alpha)} \int_{a}^{t} \psi^{\prime}(s)(\psi(t)-\psi(s))^{\alpha-1}\left|\mathcal{K}_{u_{2}}(s)\right| d s\right. \\
& +\frac{1}{\Lambda}\left\{\frac{\lambda}{\Gamma(\alpha)} \int_{a}^{\eta} \psi^{\prime}(s)(\psi(\eta)-\psi(s))^{\alpha-1}\left|\mathcal{K}_{u_{1}}(s)\right| d s\right. \\
& \left.\left.+\frac{1}{\Gamma(\alpha)} \int_{a}^{T} \psi^{\prime}(s)(\psi(T)-\psi(s))^{\alpha-1}\left|\mathcal{K}_{u_{1}}(s)\right| d s\right\}\right\} \\
= & \Lambda_{1} r_{0}+\Lambda_{2}<r_{0}
\end{aligned}
$$

which gives

$$
\left\|\mathcal{G}_{1} u_{1}+\mathcal{G}_{2} u_{2}\right\| \leq r_{0}
$$

This proves that $\mathcal{G}_{1} u_{1}+\mathcal{G}_{2} u_{2} \in \mathcal{B}_{r_{0}}$ for every $u_{1}, u_{2} \in \mathcal{B}_{r_{0}}$.

Step 2: $\mathcal{G}_{1}$ is a contration mapping on $\mathcal{B}_{n}$ since $\mathcal{G}$ is contraction mapping as in Theorem 1, then $\mathcal{G}_{1}$ is a contraction map too.

Step 3: The operator $\mathcal{G}_{2}$ is completely continuous on $\mathcal{B}_{r_{0}}$ First, from the 
continuity of $\mathcal{K}_{u}(\cdot)$, we conclude that the operator $\mathcal{G}_{2}$ is continuous. Next, It is easy to verify that

$$
\left\|\mathcal{G}_{2} u\right\| \leq \frac{L_{1} r_{0}+\mu}{1-L_{2}} \frac{(\psi(t)-\psi(s))^{\alpha}}{\Gamma(\alpha+1)}<r_{0}
$$

due to definitions of $\Lambda$ and $r_{0}$. This proves that $\mathcal{G}_{2}$ is uniformly bounded on $\mathcal{B}_{r_{0}}$ Finally, we prove that $\mathcal{G}_{2}$ maps bounded sets into equicontinuous sets of $\mathcal{C}(J, \mathbb{R})$, i.e., $\left(\mathcal{G B}_{r_{0}}\right)$ is equicontinuous. We estimate the derivative of $\mathcal{G}_{2} u(t)$

$$
\begin{aligned}
\left|\left(\mathcal{G}_{2} u\right)^{\prime}(t)\right| & =\left|\frac{1}{\Gamma(\alpha)} \int_{a}^{t} \psi^{\prime}(s)(\psi(t)-\psi(s))^{\alpha-2} \mathcal{K}_{u}(s) d s\right| \\
& \leq \frac{1}{\Gamma(\alpha)} \int_{a}^{t} \psi^{\prime}(s)(\psi(t)-\psi(s))^{\alpha-2}\left|\mathcal{K}_{u}(s)\right| d s \\
& \leq \frac{L_{1} r_{0}+\mu}{1-L_{2}} \frac{(\psi(T)-\psi(s))^{\alpha-1}}{\Gamma(\alpha+1)}:=K
\end{aligned}
$$

Now, Let $t_{1}, t_{2} \in J$, with $t_{1}<t_{2}$ and for any $u \in \mathcal{B}_{n}$.

Thus, we get

$$
\left|\mathcal{G}_{2} u\left(t_{1}\right)-\mathcal{G}_{2} u\left(t_{2}\right)\right|=\int_{t_{1}}^{t_{2}}\left|\left(\mathcal{G}_{2} u\right)^{\prime}(s)\right| d s \leq K\left(t_{2}-t_{1}\right) .
$$

From the last estimate, we deduce that

$$
\left|\mathcal{G}_{2} u\left(t_{1}\right)-\mathcal{G}_{2} u\left(t_{2}\right)\right| \rightarrow 0 \text { when } \quad t_{2} \rightarrow t_{1}, u \in \mathcal{B}_{r_{0}} .
$$

This proves that $\mathcal{G}_{2}$ is equicontinuous on $\mathcal{B}_{r_{0}}$. In view of the foregoing arguments, the Arzelá-Ascoli theorem applies and hence $\mathcal{G}_{2}$ is compact on $\mathcal{B}_{r_{0}}$. Thus, the hypothesis of Krasnoselskii fixed point theorem is fulfilled, which leads to the conclusion that there exists at least one solution on $J$. 


\section{Ulam-Hyers Stability}

In the recent section, we interested to studied UH and GUH of $\psi$ Caputo-type for the problem (11) The following observations are taken from $[24,27,9]$

Definition 4. The problem (1) is UH stable, if there exists a real number $L_{f}>0$, such that for each $\varepsilon>0$ and for every solution $\tilde{u} \in \mathcal{C}(J, \mathbb{R})$ of the inequality

$$
\left|{ }^{C} \mathcal{D}_{a^{+}}^{\alpha ; \psi} \tilde{u}(t)-f\left(t, \tilde{u}(t),{ }^{C} \mathcal{D}_{a^{+}}^{\alpha ; \psi} \tilde{u}(t)\right)\right| \leq \varepsilon, \quad t \in J
$$

there exists a unique solution $u \in \mathcal{C}(J, \mathbb{R})$ of (1) with

$$
|\tilde{u}(t)-u(t)| \leq L_{f} \varepsilon, \quad t \in J .
$$

Definition 5. The problem (11) is GUH stable if there exists $\varphi \in \mathcal{C}([0, \infty)$, $[0, \infty))$ with $\varphi(0)=0$, such that for each solution $\bar{u} \in \mathcal{C}(J, \mathbb{R})$ of the inequality

$$
\left|{ }^{C} \mathcal{D}_{a^{+}}^{\alpha ; \psi} \tilde{u}(t)-f\left(t, \tilde{u}(t),{ }^{C} \mathcal{D}_{a^{+}}^{\alpha ; \psi} \tilde{u}(t)\right)\right| \leq \varepsilon, \quad t \in J
$$

there exists a unique solution $u \in \mathcal{C}(J, \mathbb{R})$ of (1) with

$$
|\tilde{u}(t)-u(t)| \leq \varphi(\varepsilon), t \in J .
$$

Remark 1. Let $\alpha>0$. A function $\tilde{u} \in \mathcal{C}(J, \mathbb{R})$ is a solution of the inequality (11) defined by

$$
\left|{ }^{C} \mathcal{D}_{a^{+}}^{\alpha ; \psi} \tilde{u}(t)-f\left(t, \tilde{u}(t),{ }^{C} \mathcal{D}_{a^{+}}^{\alpha ; \psi} \tilde{u}(t)\right)\right| \leq \varepsilon, \quad t \in J .
$$

If and only if there exist a function $h_{\tilde{u}} \in \mathcal{C}(J, \mathbb{R})$ such that

(1) $\left|h_{\check{u}}(t)\right| \leq \varepsilon$ for all $t \in J$.

(2) $\mathcal{D}_{a^{+}}^{\alpha ; \psi} \tilde{u}(t)=f\left(t, \tilde{u}(t),{ }^{C} \mathcal{D}_{a^{+}}^{\alpha ; \psi} \tilde{u}(t)\right)+h_{\tilde{u}}(t), t \in J$.

Lemma 6. Let $\tilde{u} \in \mathcal{C}(J, \mathbb{R})$ is a solution of the inequality (11). Then $\tilde{u}$ is a solution of the following integral inequality:

$$
\left|\tilde{u}(t)-w_{\tilde{u}}-\frac{1}{\Gamma(\alpha)} \int_{a}^{t} \psi^{\prime}(s)(\psi(t)-\psi(s))^{\alpha-1} f\left(s, \tilde{u}(s),{ }^{C} \mathcal{D}_{a^{+}}^{\alpha ; \psi} \bar{u}(s)\right) d s\right|
$$




$$
\leq\left\{\frac{(\psi(T)-\psi(s))^{\alpha}}{\Gamma(\alpha+1)}+\frac{1}{\Lambda}\left\{\frac{\lambda(\psi(\eta)-\psi(s))^{\alpha}}{\Gamma(\alpha+1)}+\frac{(\psi(T)-\psi(s))^{\alpha}}{\Gamma(\alpha+1)}\right\}\right\} \varepsilon
$$

where

$$
\begin{aligned}
w_{\tilde{u}}= & \left\{\frac { 1 } { \Lambda } \left\{\frac{\lambda}{\Gamma(\alpha)} \int_{a}^{\eta} \psi^{\prime}(s)(\psi(\eta)-\psi(s))^{\alpha-1} \mathcal{K}_{u}(s) d s\right.\right. \\
& \left.\left.+\frac{1}{\Gamma(\alpha)} \int_{a}^{T} \psi^{\prime}(s)(\psi(T)-\psi(s))^{\alpha-1} \mathcal{K}_{u}(s) d s\right\}\right\} .
\end{aligned}
$$

Proof. In view of Remark 1 and Theorem 1, we obtain

$$
\begin{aligned}
& \widetilde{u}(t)=\frac{1}{\Gamma(\alpha)} \int_{a}^{t} \psi^{\prime}(s)(\psi(t)-\psi(s))^{\alpha-1}\left[f\left(s, \widetilde{u}(s),{ }^{C} \mathcal{D}_{a^{+}}^{\alpha ; \psi} \widetilde{u}(s)\right)+h_{\widetilde{u}}(s)\right] d s \\
& +\left\{\frac { 1 } { \Lambda } \left\{\frac{\lambda}{\Gamma(\alpha)} \int_{a}^{\eta} \psi^{\prime}(s)(\psi(\eta)-\psi(s))^{\alpha-1}\left[f\left(s, \widetilde{u}(s),{ }^{C} \mathcal{D}_{a^{+}}^{\alpha ; \psi} \widetilde{u}(s)\right)+h_{\widetilde{u}}(s)\right] d s\right.\right. \\
& \left.\left.-\frac{1}{\Gamma(\alpha)} \int_{a}^{T} \psi^{\prime}(s)(\psi(T)-\psi(s))^{\alpha-1}\left[f\left(s, \widetilde{u}(s),{ }^{C} \mathcal{D}_{a^{+}}^{\alpha ; \psi} \widetilde{u}(s)\right)+h_{\widetilde{u}}(s)\right] d s\right\}\right\} .
\end{aligned}
$$

It follows that

$$
\begin{aligned}
& \mid \tilde{u}(t)-w_{\tilde{u}}-\frac{1}{\Gamma(\alpha)} \int_{a}^{t} \psi^{\prime}(s)(\psi(t)-\psi(s))^{\alpha-1} f\left(s, \tilde{u}(s),{ }^{C} \mathcal{D}_{a^{+}}^{\alpha ; \psi} \tilde{u}(s)\right) d s \mid \\
& \leq \frac{1}{\Gamma(\alpha)} \int_{a}^{t} \psi^{\prime}(s)(\psi(t)-\psi(s))^{\alpha-1}\left|h_{\widetilde{u}}(s)\right| d s \\
&+\left\{\frac { 1 } { \Lambda } \left\{\frac{\lambda}{\Gamma(\alpha)} \int_{a}^{\eta} \psi^{\prime}(s)(\psi(\eta)-\psi(s))^{\alpha-1}\left|h_{\widetilde{u}}(s)\right| d s\right.\right. \\
&\left.\left.+\frac{1}{\Gamma(\alpha)} \int_{a}^{T} \psi^{\prime}(s)(\psi(T)-\psi(s))^{\alpha-1}\left|h_{\widetilde{u}}(s)\right| d s\right\}\right\} \\
& \leq \frac{\varepsilon}{\Gamma(\alpha)} \int_{a}^{t} \psi^{\prime}(s)(\psi(t)-\psi(s))^{\alpha-1} d s \\
&+\left\{\frac { \varepsilon } { \Lambda } \left\{\frac{\lambda}{\Gamma(\alpha)} \int_{a}^{\eta} \psi^{\prime}(s)(\psi(\eta)-\psi(s))^{\alpha-1} d s\right.\right. \\
&\left.\left.+\frac{1}{\Gamma(\alpha)} \int_{a}^{T} \psi^{\prime}(s)(\psi(T)-\psi(s))^{\alpha-1} d s\right\}\right\} \\
& \leq\left\{\frac{(\psi(T)-\psi(s))^{\alpha}}{\Gamma(\alpha+1)}+\frac{1}{\Lambda}\left\{\frac{\lambda(\psi(\eta)-\psi(s))^{\alpha}}{\Gamma(\alpha+1)}+\frac{(\psi(T)-\psi(s))^{\alpha}}{\Gamma(\alpha+1)}\right\}\right\}
\end{aligned}
$$


Theorem 3. If hypotheses of Theorem 1 are fulfilled. Then the problem (1) is Ulam-Hyers stable.

Proof. Let $\varepsilon>0$, and $\tilde{u} \in \mathcal{C}(J, \mathbb{R})$ be a function which satisfies the inequality (11), and let $u \in \mathcal{C}(J, \mathbb{R})$ be the unique solution of the following $\psi$-Caputo fractional differential equation

$$
{ }^{C} \mathcal{D}_{a^{+}}^{\alpha ; \rho} u(t)=f\left(t, u(t),{ }^{C} \mathcal{D}_{a^{+}}^{\alpha \rho} u(t)\right), \quad t \in J
$$

with

$$
u(\eta)=\tilde{u}(\eta), u(T)=\tilde{u}(T)
$$

where $0<\alpha<1$. Using Lemma 4, It is easily seen that $u(\cdot)$ satisfies the integral equation

$$
u(t)=w_{u}+\frac{1}{\Gamma(\alpha)} \int_{a}^{t} \psi^{\prime}(s)(\psi(t)-\psi(s))^{\alpha-1} f\left(s, u(s),{ }^{C} \mathcal{D}_{a^{+}}^{\alpha ; \psi} u(s)\right) d s
$$

where

$$
\begin{aligned}
w_{u}=\{ & \frac{1}{\Lambda}\left\{\frac{\lambda}{\Gamma(\alpha)} \int_{a}^{\eta} \psi^{\prime}(s)(\psi(\eta)-\psi(s))^{\alpha-1} f\left(s, u(s),{ }^{C} \mathcal{D}_{a^{+}}^{\alpha ; \psi} u(s)\right) d s\right. \\
& \left.\left.+\frac{1}{\Gamma(\alpha)} \int_{a}^{T} \psi^{\prime}(s)(\psi(T)-\psi(s))^{\alpha-1} f\left(s, u(s),{ }^{C} \mathcal{D}_{a^{+}}^{\alpha ; \psi} u(s)\right) d s\right\}\right\} .
\end{aligned}
$$

Applying Lemma 6, we obtain

$$
\left|\tilde{u}(t)-w_{\tilde{u}}-\frac{1}{\Gamma(\alpha)} \int_{a}^{t} \psi^{\prime}(s)(\psi(t)-\psi(s))^{\alpha-1} f\left(s, \tilde{u}(s),{ }^{C} \mathcal{D}_{a^{+}}^{\alpha ; \psi} \tilde{u}(s)\right) d s\right| \leq V \varepsilon
$$

where

$$
V:=\left\{\frac{(\psi(T)-\psi(s))^{\alpha}}{\Gamma(\alpha+1)}+\frac{1}{\Lambda}\left\{\frac{\lambda(\psi(\eta)-\psi(s))^{\alpha}}{\Gamma(\alpha+1)}+\frac{(\psi(T)-\psi(s))^{\alpha}}{\Gamma(\alpha+1)}\right\}\right\} .
$$

From (17) we can easily get that $\left|w_{\tilde{u}}-w_{u}\right| \rightarrow 0$. Indeed, from (H1)and (17), we obtain that

$$
\left|w_{\tilde{u}}-w_{u}\right|=\mid\left\{\frac { 1 } { \Lambda } \left\{\frac{\lambda}{\Gamma(\alpha)} \int_{a}^{\eta} \psi^{\prime}(s)(\psi(\eta)-\psi(s))^{\alpha-1} f\left(s, \tilde{u}(\tau),{ }^{C} \mathcal{D}_{a^{+}}^{\alpha ; \psi} \tilde{u}(s)\right) d s\right.\right.
$$




$$
\begin{aligned}
& \left.\left.-\frac{1}{\Gamma(\alpha)} \int_{a}^{T} \psi^{\prime}(s)(\psi(T)-\psi(s))^{\alpha-1} f\left(s, \tilde{u}(\tau),{ }^{C} \mathcal{D}_{a^{+}}^{\alpha ; \psi} \tilde{u}(s)\right) d s\right\}\right\} \\
& -\left\{\frac { 1 } { \Lambda } \left\{\frac{\lambda}{\Gamma(\alpha)} \int_{a}^{\eta} \psi^{\prime}(s)(\psi(\eta)-\psi(s))^{\alpha-1} f\left(s, u(s),{ }^{C} \mathcal{D}_{a^{+}}^{\alpha} u(s)\right) d s\right.\right. \\
& \left.\left.+\frac{1}{\Gamma(\alpha)} \int_{a}^{T} \psi^{\prime}(s)(\psi(T)-\psi(s))^{\alpha-1} f\left(s, u(s),{ }^{C} \mathcal{D}_{a^{+}}^{\alpha} u(s)\right) d s\right\}\right\} \mid \\
\leq & \frac{1}{\Lambda}\left\{\lambda \mathcal{I}_{a^{+}}^{\alpha ; \psi}\left|f\left(\eta, \widetilde{u}(\eta),{ }^{C} \mathcal{D}_{a^{+}}^{\alpha ; \psi} \widetilde{u}(\eta)\right)-f\left(\eta, u(\eta),{ }^{C} \mathcal{D}_{a^{+}}^{\alpha ; \psi} u(\eta)\right)\right|\right. \\
& \left.+\mathcal{I}_{a^{+}}^{\alpha ; \psi}\left|f\left(T, \widetilde{u}(T),{ }^{C} \mathcal{D}_{a^{+}}^{\alpha, \rho} \widetilde{u}(T)\right)-f\left(T, u(T),{ }^{C} \mathcal{D}_{a^{+}}^{\alpha ; \psi} u(T)\right)\right|\right\}
\end{aligned}
$$

since,

$$
\begin{aligned}
& \left|f\left(T, \widetilde{u}(T),{ }^{C} \mathcal{D}_{a^{+}}^{\alpha \rho} \widetilde{u}(T)\right)-f\left(T, u(T),{ }^{C} \mathcal{D}_{a^{+}}^{\alpha \rho} u(T)\right)\right| \\
& \quad \leq L_{1}|u(T)-u(T)|+L_{2} \mid{ }^{C} \mathcal{D}_{a^{+}}^{\alpha, \rho} \tilde{u}(T)-{ }^{C} \mathcal{D}_{a^{+}}^{\alpha \rho} u(T) \\
& \quad \leq \frac{L_{1}}{1-L_{2}}|\widetilde{u}(T)-u(T)| .
\end{aligned}
$$

Similarly, we obtain

$$
\left|f\left(\eta, \widetilde{u}(\eta),{ }^{C} \mathcal{D}_{a^{+}}^{\alpha \rho} \widetilde{u}(\eta)\right)-f\left(\eta, u(\eta),{ }^{C} \mathcal{D}_{a^{+}}^{\alpha \rho} u(T)\right)\right| \leq \frac{L_{1}}{1-L_{2}}|\widetilde{u}(\eta)-u(\eta)|
$$

which implies

$$
\left|w_{\tilde{u}}-w_{u}\right| \leq \frac{L_{1}}{\left(1-L_{2}\right)} \frac{1}{\Lambda}\left\{\lambda \mathcal{I}_{a^{+}}^{\alpha \rho}|\tilde{u}(\eta)-u(\eta)|+\mathcal{I}_{a^{+}}^{\alpha \rho}|\tilde{u}(\eta)-u(\eta)|\right\} \rightarrow 0 .
$$

Hence,

$$
u(t)=w_{u}+\frac{1}{\Gamma(\alpha)} \int_{a}^{t} \psi^{\prime}(s)(\psi(t)-\psi(s))^{\alpha-1} f\left(s, u(s),{ }^{C} \mathcal{D}_{a^{+}}^{\alpha ; \psi} u(s)\right) d s .
$$

According to (18), (H1) and (19), we obtain

$$
\begin{aligned}
& |\tilde{u}(t)-u(t)| \\
& \leq\left|\tilde{u}(t)-w_{\tilde{u}}-\frac{1}{\Gamma(\alpha)} \int_{a}^{t} \psi^{\prime}(s)(\psi(t)-\psi(s))^{\alpha-1} f\left(s, \tilde{u}(s),{ }^{C} \mathcal{D}_{a^{+}}^{\alpha ; \psi} \tilde{u}(s)\right) d s\right| \\
& \quad+\frac{1}{\Gamma(\alpha)} \int_{a}^{t} \psi^{\prime}(s)(\psi(t)-\psi(s))^{\alpha-1} \mid f\left(s, \tilde{u}(s),{ }^{C} \mathcal{D}_{a^{+}}^{\alpha ; \psi} \tilde{u}(s)\right) \\
& \quad-f\left(s, u(s),{ }^{C} \mathcal{D}_{a^{+}}^{\alpha ; \psi} u(s)\right) \mid d s
\end{aligned}
$$




$$
\leq V \varepsilon+\frac{L_{1}}{1-L_{2}} \frac{1}{\Gamma(\alpha)} \int_{a}^{t} \psi^{\prime}(s)(\psi(t)-\psi(s))^{\alpha-1}|\widetilde{u}(s)-u(s)| d s .
$$

By Lemma 2, there exists a constant $L_{f}>0$ independent of $\varepsilon$ such that

$$
|\tilde{u}(t)-u(t)| \leq L_{f} \varepsilon
$$

Therefore the problem (1) is Ulam-Hyers stable.

Corollary 1. Under assumptions of Theorem 3 , Assume that $\varphi: \mathbb{R}^{+} \rightarrow \mathbb{R}^{+}$ such that $\varphi(0)=0$. Then tne problem (41) is generalized Ulam-Hyers stable.

Proof. One can repeat the same processes in Theorem 3 with putting $L_{f} \varepsilon=\varphi(\varepsilon)$, and $\varphi(0)=0$, we conclude that

$$
|\tilde{u}(t)-u(t)| \leq \varphi(\varepsilon) .
$$

\section{Example}

This section is devoted to the illustration of the results derived in the last section.

Example 1. Consider the following problem of implicit fractional differential equations involving $\psi$-Caputo type:

$$
\left\{\begin{aligned}
\mathcal{D}_{0^{+}}^{\frac{1}{4} ; t} u(t) & =\left[\frac{1}{3} e^{\sqrt{t}+1}+\frac{2+|u(t)|+\left|\mathcal{D}_{0^{+}}^{\frac{1}{2} ; t} u(t)\right|}{8 e^{2-t}\left(1+|u(t)|+\left|\mathcal{D}_{0^{+}}^{\frac{1}{2} ; t} u(t)\right|\right)}\right], t \in[0,1] \\
u(T) & =\lambda u(\eta) .
\end{aligned}\right.
$$

Where

$$
\alpha=\frac{1}{4}, \lambda=\frac{3}{4}, \eta=\frac{1}{2}, a=0, T=1, \psi(t)=t,
$$

Set:

$$
f(t, u, v)=\left[\frac{1}{3} e^{\sqrt{t+1}}+\frac{2+u+v}{8 e^{2-t}(1+u+v)}\right], t \in[0,1], u, v \in \mathbb{R}^{+}
$$


Clearly, the function $f \in C([0,1])$. For each $u_{1}, v_{1}, u_{2}, v_{2} \in \mathbb{R}^{+}$and $t \in[0,1]$

$$
\begin{aligned}
\left|f(t, u, v)-f\left(t, u_{1}, v_{1}\right)\right| & =\left|\frac{2+u_{1}+v_{1}}{8 e^{2-t}\left(1+u_{1}+v_{1}\right)}-\frac{2+u_{2}+v_{2}}{8 e^{2-t}\left(1+u_{2}+v_{2}\right)}\right| \\
& \leq \frac{1}{8 e^{2-t}}\left(\left|u_{1}-u_{2}\right|+\left|v_{1}-v_{2}\right|\right) \\
& \leq \frac{1}{8 e}\left(\left|u_{1}-u_{2}\right|+\left|v_{1}-v_{2}\right|\right) .
\end{aligned}
$$

Hence, the condition (H1) is satisfied with $L_{1}=L_{2}=\frac{1}{8 e}$. It is easy to verify that

$$
\Lambda_{1}=\frac{L_{1}}{1-L_{2}}\left\{\frac{1}{\Gamma(\alpha+1)}+\frac{1}{\Lambda}\left\{\frac{\lambda \eta^{\alpha}}{\Gamma(\alpha+1)}+\frac{1}{\Gamma(\alpha+1)}\right\}\right\}<1 .
$$

Clearly, the hypothesis of Theorem 1 are fulfilled and hence its conclusion implies the existence of a unique solution of the problem in Equation (21) on $[0,1]$.

\section{References}

1. S. Abbas, M. Benchohra and G. M. N'Gurékata, Topics in Fractional Differential Equations; Springer: New York, NY, USA, 2015.

2. M. S. Abdo, S. K. Panchal and A. M. Saeed, Fractional boundary value problem with $\psi$-Caputo fractional derivative, Proc. Math. Sci., 129 (2019), 14.

3. R. P. Agarwal, M. Benchohra and S. Hamani, A survey onexistence results for boundary value problems of nonlinear fractional differential equations and inclusions, Acta Appl. Math., 109 (2010), 973-1033.

4. R. Almeida, A Caputo fractional derivative of a function with respect to another function, Commun. Nonlinear Sci., 44 (2017), 460-481.

5. R. Almeida, Fractional Differential Equations with Mixed Boundary Conditions, Bull. Malays. Math. Sci. Soc., 42 (2019), 1687-1697.

6. R. Almeida, A. B. Malinowska and M. T. T. Monteiro, Fractional differential equations with a Caputo derivative with respect to a kernel function and their applications, Math. Meth. Appl. Sci., 41 (2018), 41, 336-352.

7. R. Almeida, M. Jleli and B. Samet, A numerical study of fractional relaxationoscillation equations involving $\psi$-Caputo fractional derivative, Rev. R. Acad. Cienc. Exactas Fis. Nat. Ser. A Mat. RACSAM 113 (2019), 1873-1891.

8. M. Benchohra, J. R. Graef and S. Hamani, Existence results for boundary value problems with non-linear fractional differential equations, Appl. Anal., 87 (2008), 851-863. 
9. M. Benchohra and S. Bouriah, Existence and stability results for nonlinear boundary value problem for implicit differential equations of fractional order, Moroccan J. Pure Appl. Anal., 1 (2015), 22-37.

10. M. Benchohra, Z. Bouteffal, J. Henderson, et al., Measure of noncompactness and fractional integro-differential equations with state-dependent nonlocal conditions in Fréchet spaces, AIMS Mathematics, 5 (2020), 15-25.

11. A. Boutiara, M. S. ABDO and M. Benbachir, Existence results for $\psi$-Caputo fractional neutral functional integro-differential equations with finite delay, Turk J. Math., 44 (2020), 2380-2401.

12. A. Boutiara, K. Guerbati and M. Benbachir, Caputo-Hadamard fractional differential equation with three-point boundary conditions in Banach spaces, AIMS Mathematics, 5 (2020), No.1, 259-272.

13. A. Boutiara, M. Benbachir and K. Guerbati, Measure Of Noncompactness for Nonlinear Hilfer Fractional Differential Equation in Banach Spaces, Ikonion Journal of Mathematics, 1 (2019), No.2.

14. A. Boutiara, M. Benbachir and K. Guerbati, Caputo Type Fractional Differential Equation with Nonlocal Erdélyi-Kober Type Integral Boundary Conditions in Banach Spaces, Surveys in Mathematics and its Applications, 15 (2020), 399-418.

15. D. Chergui, T. E. Oussaeif and M. Ahcene, Existence and uniqueness of solutions for nonlinear fractional differential equations depending on lower-order derivative with non-separated type integral boundary conditions, AIMS Mathematics, 4 (2019), 112133.

16. EM. Elsayed, On the existence and stability of solution of boundary value problem for fractional integro-differential equations with complex order, Filomat. 32 (2018), No.8.

17. R. Hilfer, Applications of Fractional Calculus in Physics, World Scientific: Singapore, 2000 .

18. D. Hyers, On the stability of the linear functional equation. Proc Natl Acad Sci., 27 (1941), 222-224.

19. D. H. Hyers, G. Isac and T. M. Rassias, Stability of Functional Equations in Several Variables, Birkhuser, Boston, 1998.

20. R. W. Ibrahim, Generalized Ulam-Hyers stability for fractional differential equations, Int. J. Math., 23 (2012), No.5, 9 pages.

21. S. M. Jung, Hyers-Ulam stability of linear differential equations of first order, Appl. Math. Lett., 19 (2006), 854-858.

22. A. A. Kilbas, H. M. Srivastava and J. J. Trujillo, Theory and Applications of Fractional Differential Equations; vol. 204 of North-Holland Mathematics Sudies; Elsevier Science B.V.: Amsterdam, The Netherlands, 2006.

23. K. S. Miller and B. Ross, An Introduction to Fractional Calculus and Fractional Differential Equations, Wiley: New York, NY, USA, 1993.

24. M. Obloza, Hyers stability of the linear differential equation, Rocznik Nauk-Dydakt Prace Mat., 13 (1993), 259-270. 
25. K. B. Oldham, Fractional differential equations in electrochemistry, Adv. Eng. Softw., 41 (2010), 9-12.

26. I. Podlubny, Fractional Differential Equations; Academic Press: San Diego, CA, USA, 1999.

27. I. A. Rus, Ulam stabilities of ordinary differential equations in a Banach space, Carpath. J. Math., 26 (2010), 103-107.

28. J. Sabatier, O. P. Agrawal and J. A. T. Machado, Advances in Fractional CalculusTheoretical Developments and Applications in Physics and Engineering, Springer: Dordrecht, The Netherlands, 2007.

29. B. Samet and H. Aydi, Lyapunov-type inequalities for an anti-periodic fractional boundary value problem involving $\psi$-Caputo fractional derivative, J. Inequal. Appl., 2018, 286.

30. K. Shah, W. Hussain, P. Thounthong, et al., On nonlinear implicit fractional differential equations with integral boundary condition involving $p$-Laplacian operator without compactness, Thai J. Math., 16 (2018), 301-321.

31. V. E. Tarasov, Fractional Dynamics: Application of Fractional Calculus to Dynamics of Particles, Fields and Media; Springer Science \& Business Media: Berlin/Heidelberg, Germany, 2010.

32. S. M. Ulam, A Collection of the Mathematical Problems, Interscience, New York (1960)

33. Y. Zhou, Basic Theory of Fractional Differential Equations; World Scientific: Singapore, 2014. 